\title{
The Design and Analysis of Invisible Radome with Sandwich-like Properties
}

\author{
Tianzhen MENG, Weiwei WU, Naichang YUAN \\ Dept. of Electronic Science and Engineering, National University of Defense Technology, \\ Deya Street 109, 61200 Changsha, China \\ mengtianzhen15@nudt.edu.cn, \{wwei_wu,nchang_yuan\}@sina.com
}

Submitted October 27, 2016 / Accepted March 24, 2017

\begin{abstract}
A novel thin radome made by metamaterial is presented, with the properties like the sandwich where the middle acts as a pass band filter at an expected frequency band, while others behave as absorbers. This new radome consists of three layers including metal goblets-shape the frequency selective surface (FSS) layer, a substrate and a resistive square loops. Compared with the traditional invisible radome, the new radome's two absorptive bands were separated by one transmission band, which is a challenge for the traditional ones. Except for the analysis of absorption property, the effects of the radome for the antenna are also simulated and some useful conclusions are drawn. Also, it has the advantages of simple structure for the easy fabrication, which could be more attracting in practical radome.
\end{abstract}

\section{Keywords}

Invisible radome, $\mathrm{RCS}$ reduction, resistive material

\section{Introduction}

Frequency selective surface (FSS), a periodic artificially synthesized material, shows different reflection or transmission properties for incident waves with different frequencies. The American AirForce utilized such configuration, where electromagnetic waves out of the operating frequency will be reflected to other directions. However, this is only effective for monostatic radar to reduce the radar cross section (RCS). The technology of absorptive/ transmitted FSS (A/T FSS), which employed metal FSS and absorptive material, gave a new solution to RCS reduction.

The first A/T FSS structure was proposed in 1995 in a patent in the United States [1]. The relative reports have come out progressively in the last few years. In 2012, Costa Filippo designed a radome with wideband absorbing properties [2], which was realized by placing a resistive FSS on top of an interdigitated Jerusalem cross metallic FSS. In addition, an alternative approach was given to evaluate transmission and reflection properties of FSSs through a simple and accurate first-order circuit [3], [4]. In the last three years, the technologies on RCS reduction can be summarized as follows: wide band absorber [5-7], absorptive FSS with magnetic material [8-10], absorptive metamaterial without considering transmission properties [8], [9], and new A/T FSS structure [10-12]. The common lies in that the absorption band is higher than the transmission one while fewer studies are reported on the contrary case. Nevertheless, A/T FSS with sandwich-like properties, that two frequency bands show absorption property while another one between the absorptive bands shows transmissive, is the favorite for radar stealth. The band-pass A/T FSS [12] realized by lump resistance is complex for fabrication, and its absorptive band is narrow. To gain the better performance and easy fabrication, a $5 \mathrm{~mm}$-thick three-layer $\mathrm{A} / \mathrm{T}$ FSS radome is proposed. The lossy materials of resistance film are employed for its good bilateral absorbing properties while a new metal FSS are designed. The results by simulations are analyzed and absorbing mechanism is also drawn.

This paper is organized as follows. The structure of our new A/T FSS were described in the following section. In Sec. 3, the design and analysis of the single part including the pass-band metal FSS, absorptive FSS and the whole A/T FSS is presented. In addition we summarized the design principles. In Sec. 4, the whole system constructed by an antenna and the corresponding invisible radome were studied. The influence of radome on the gain and RCS of the antenna was addressed followed by concluding remarks.

\section{Description of the Structure}

Our target antenna is a waveguide slot antenna with operating band around $15 \mathrm{GHz}$. The main goal of our new design is to obtain two absorption wide bands, in the middle of which there is one wide-band polarization-sensitive transmission band around $15 \mathrm{GHz}$. In this design, the unit cell has three layers, comprising the dielectric substrate of Taconic's TSM-DS3 material which has a relative permittivity of 3.0 and dielectric loss tangent of 0.0011 . In metal plane, to obtain the transmission properties for the FSSs, 


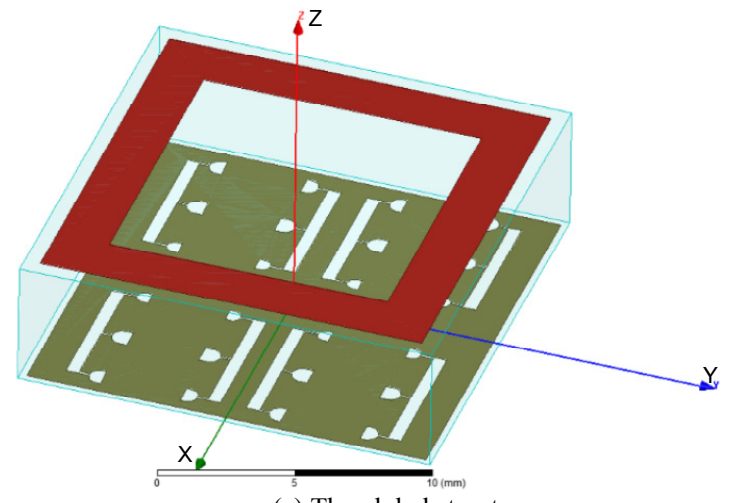

(a) The global structure
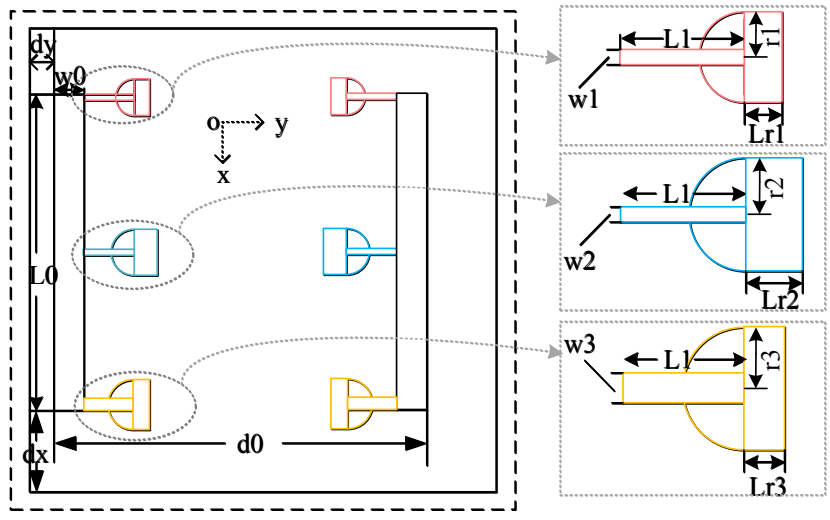

(b) The sketch of one quarter of the metal element

Fig. 1. The configuration of our FSS unit.

\begin{tabular}{|c|c|}
\hline Name & Dimension (unit: $\mathbf{~ m m )}$ \\
\hline w0 & 0.5 \\
\hline L0 & 5.2 \\
\hline L1 & 0.8 \\
\hline w1 & 0.1 \\
\hline r1 & 0.3 \\
\hline Lr1 & 0.2 \\
\hline w2 & 0.1 \\
\hline 2 2 & 0.4 \\
\hline Lr2 & 0.3 \\
\hline w3 & 0.2 \\
\hline r3 & 0.4 \\
\hline Lr3 & 0.15 \\
\hline d0 & 6 \\
\hline dx & 1.65 \\
\hline dy & 0.5 \\
\hline
\end{tabular}

Tab. 1. The optimized dimensions of the unit cell.

rectangles and semicircles were united looking like goblet shelves. FSS units of goblets slot etched in metal plane (metallic foil) stick to the bottom side, and square resistance film loops stick to the top side. That two periodic structures are realized by etching process on the surface of the dielectric substrate. The structure of unit cell is shown in Fig. 1(a), where the red part is the resistance layer, the bronze part is the metal layer, and the translucent green part is the substrate. The sketch of one quarter of the metal element is given in Fig. 1(b) to describe the geometry of our goblet.

The thickness of the dielectric substrate is $5 \mathrm{~mm}$. The upper layer is made up of the resistance film(50 $\Omega / \mathrm{sq})$ unit, which is a loss material, in the shape of a rectangular ring, with its width being $2 \mathrm{~mm}$. The length of the outer side is $14.8 \mathrm{~mm}$ while the length of the inner side is $10.8 \mathrm{~mm}$.

As to the metal FSS layer, each unit contains four pairs of goblet-shelf shaped slots which are both symmetric along the $\mathrm{X}$-axis and $\mathrm{Y}$-axis. Each pair of goblet-shelves are bilateral symmetric. The outline structure is one quarter of the metal unit. L0 is the length of the big rectangles and $\mathrm{d} 0$ is the distance of the two big rectangles. Each goblet is split into two small rectangles and one semicircle. More accurately, the goblets are zoomed out on the right part of Fig. 1(b). The optimized parameters are given in Tab. 1.

\section{Design and Analysis of Our Metamaterial}

To analyze the performance of the radome, the main parameters including S11, S22, S21 and S12 are simulated by using a commercial full-wave FEM simulator named Ansoft HFSS, which can assign the master and slave boundary to obtain the results of the periodic structure just by simulating single unit cell.

\subsection{Resistive FSS Layer}

Traditional Salisbury absorber places a resistance layer in front of grounded layer with a distance of a $1 / 4$ wavelength, which is the central frequency of absorptionband. Replacing the resistance plate by periodic lossy material surface, the central frequency of absorption-band can keep unchanged. Moreover, the absorbing properties can adjust by varying the parameters of the unit cell. The relationship between reflection coefficient and frequency is shown in Fig. 2. Using the resonant periodic structure depicted in Fig. 1, the configuration with bilateral absorbing characteristics can be done. To ensure the pass-band of the operating frequency is not affected, several principles need to be met as follows:

(1) The metal FSS should reflect the whole electromagnetic wave with the frequency of $f_{2}$, when the resistance layer is substituted for the PEC.

(2) When there is no reflection ground layer, the resistance layer absorption of electromagnetic wave with the frequency of $f_{2}$ is little.

(3) With reflection ground layer, the reflection coefficient of the resistance layer is close to $0 \mathrm{~dB}$ around $f_{2}$ which is mainly up to the parameters: the slab thickness of $d$. At the same time the reflection coefficients are all below than $-10 \mathrm{~dB}$ near $f_{1}$ and $f_{3}$, which shows good absorption property, where main factors are structure shape and size.

In this design, $f_{2}$ is $15 \mathrm{GHz}, f_{1}=f_{2} / 2=7.5 \mathrm{GHz}$, and the relative permittivity of substrate is 3.0. The relationship between frequency and permittivity is $f_{c 0}=f_{0} / \sqrt{\varepsilon_{r}}$, then, the thickness of substrate can be calculated as $d=\lambda_{1} /\left(4 \sqrt{ } \overline{\varepsilon_{\mathrm{r}}}\right)$ $=5.77 \mathrm{~mm}$. 


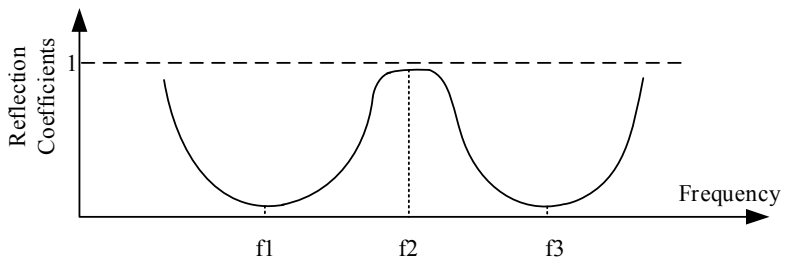

Fig. 2. The relationship between reflection coefficient and frequency of absorber.

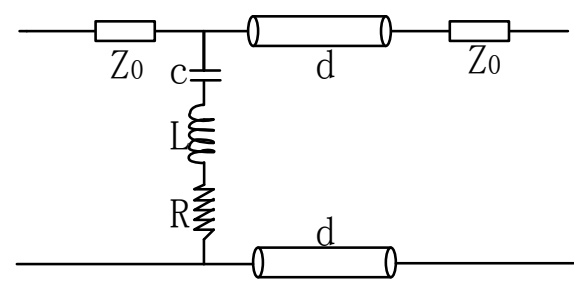

Fig. 3. The equivalent circuit of resistance unit cell.

To pay attention to the absorbing characteristics, $d$ is set to $5 \mathrm{~mm}$. Resistive periodic structure is the classical ring square loop, attached to the dielectric substrate Taconic's TSM-DS3. The equivalent circuit is shown in Fig. 3.

The transmission coefficients of the resistance layer substituted for the PEC is shown in Fig. 4(a). It is a bandstop filter, which has good transmission property around $15 \mathrm{GHz}$, meeting the first principle above. Fig. 4(b) gives the reflection coefficients varying with frequencies of the resistance layer with ground layer. The $\mathrm{S} 11$ around $15 \mathrm{GHz}$ approximates $0 \mathrm{~dB}$, which means all the electromagnetic

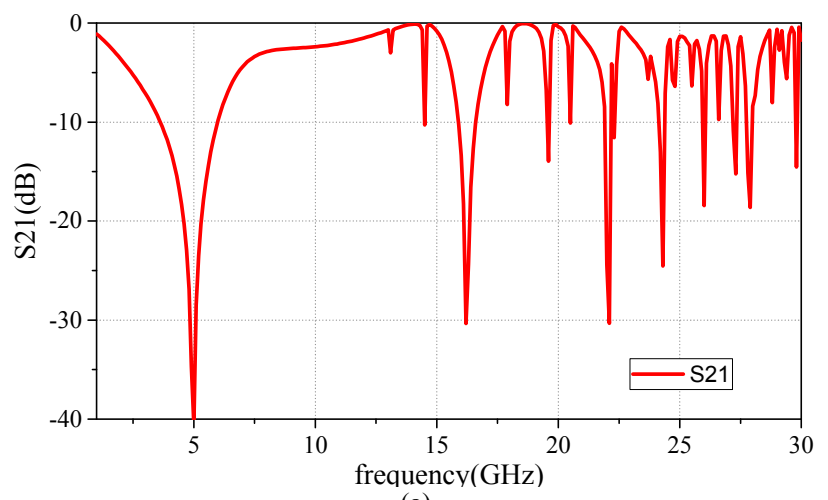

(a)

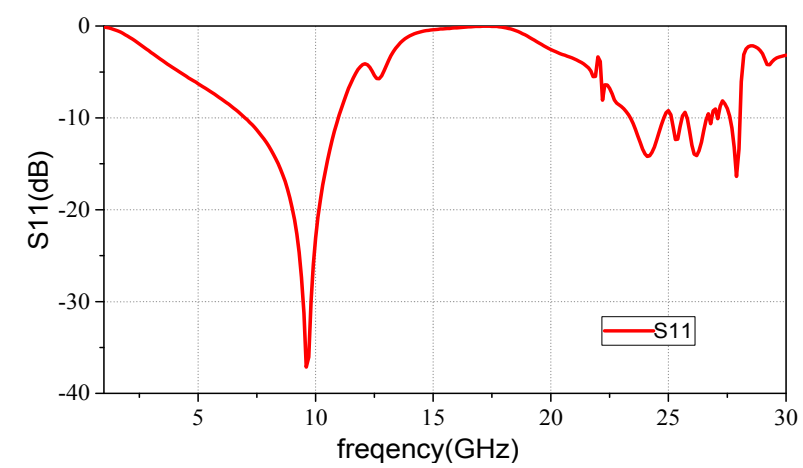

(b)

Fig. 4. (a) The transmission coefficients of square loop of PEC; (b) the reflection coefficients of square loop of resistance. wave energy is reflected, no absorption happen from $14 \mathrm{GHz}$ to $18 \mathrm{GHz}$, satisfying the second principle above. Also, from $5.6 \mathrm{GHz}$ to $11 \mathrm{GHz}$ and from $23.2 \mathrm{GHz}$ to $27.8 \mathrm{GHz}, \mathrm{S} 11$ is below $-10 \mathrm{~dB}$. Since there is a ground layer, through which no electromagnetic waves pass. The absorption rate of these two bands is more than $90 \%$, the third principle is satisfied as well.

\subsection{Passband Metal FSS Layer}

The metal FSS should have the following characteristics: Firstly, good transmission properties in the vicinity of $f_{2}$, in order to ensure the bandpass ability. Secondly, good reflection characteristics in the vicinity of $f_{1}$ and $f_{3}$, to ensure the absorbing effect of resistance layer and implement bilateral absorbing ability. The ratio of the metal element period to the impedance surface period should be a positive integer. Thus, considering the period size and the bandpass property, a slightly complicated stricter, goblets structure, is selected. Each slot structure consists of a big vertical rectangles and three goblets. Each unit has 2 slots. The equivalent circuit is depicted in Fig. 5.

The length of the big rectangle L0 mainly controls the resonant frequency. The transmission coefficients varying with frequency for different rectangle length is shown in Fig. 6. The resonant frequency is higher when L0 is smaller. For the design target is $15 \mathrm{GHz}$, we choose $\mathrm{L} 0=5.3 \mathrm{~mm}$.

The goblets added to the big rectangles contribute to fine-tuning of the frequency response and the miniaturization of the structure. It is useful when the three layer are combined. Since the two resonant frequencies are close to each other, they show a wider pass band and steeper dropping characteristics. The distance between the two big

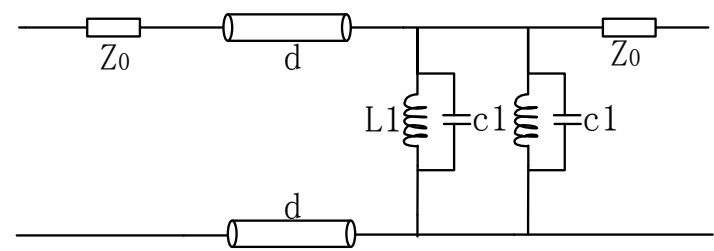

Fig. 5. The equivalent circuit of metal FSS unit cell.

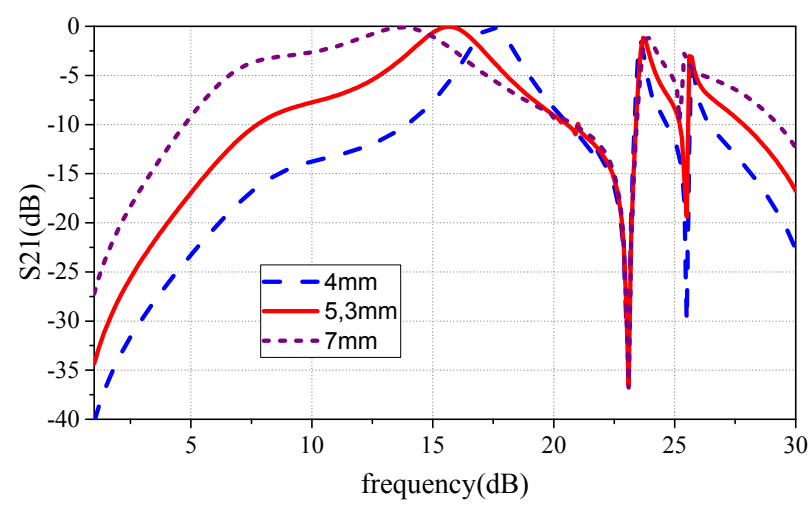

Fig. 6. The frequency response changing with the LO. 


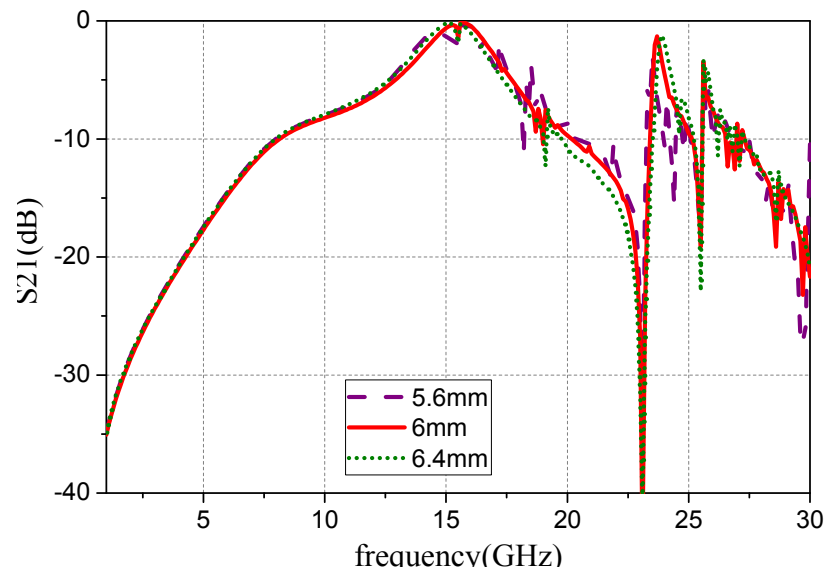

Fig. 7. The frequency response changing with the d0.

rectangles d0 determines the two close resonant frequencies, which can control the bandwidth and transmission property of transmission band. The frequency response changing with the $\mathrm{d} 0$ is shown in Fig. 7. It can be seen that $\mathrm{d} 0=6 \mathrm{~mm}$ is an appropriate value.

The period length of the metal FSS layer is half of the period length of the resistance layer. When combining with the three layers, a unit contains four metal FSS unit. For good transmission property, the slots were moved toward the center of the unit. And a gap was added between different units which is equivalent to a series LC circuits. The different frequency response of metal FSS layer is shown in Fig. 8.

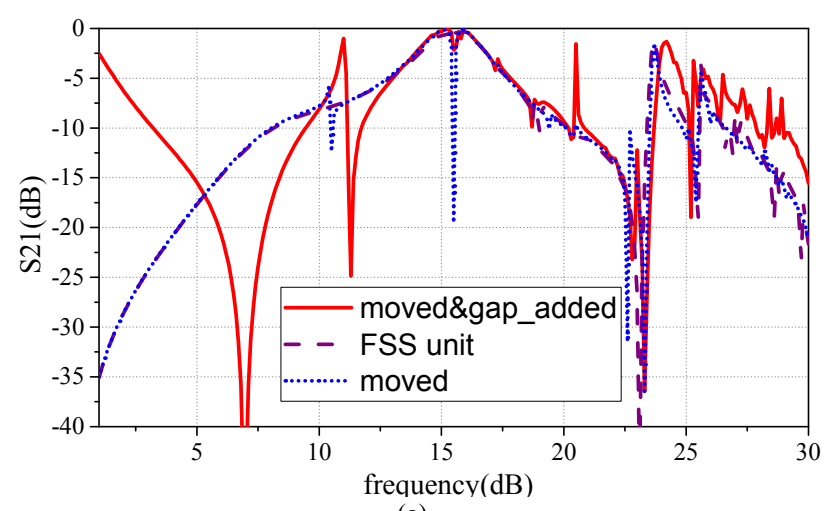

(a)

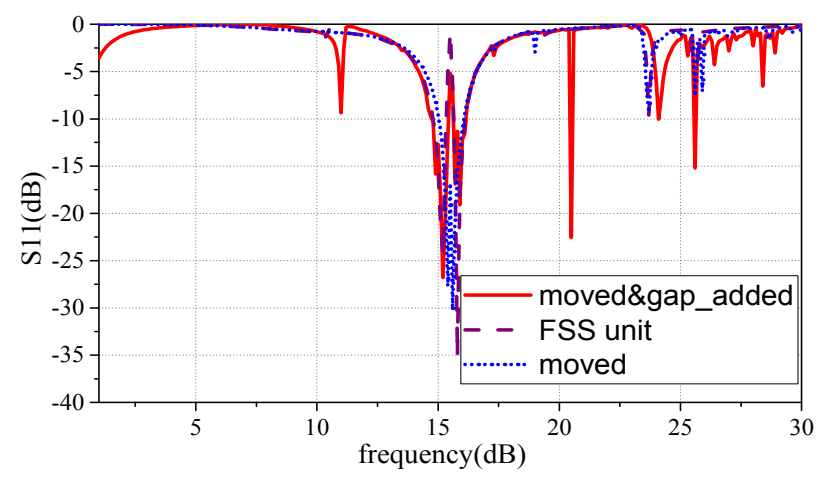

(b)

Fig. 8. The transmission coefficients (a) and reflection coefficients (b) of the metal FSS layer.

\subsection{Transmission and Reflection Parameters of A/T FSS}

The designed radome was simulated and the results of the transmission and reflection coefficients were shown in Fig. 9.

It can be seen from Fig. 9(a) that S21 presents the band-pass characteristic. From $14.3 \mathrm{GHz}$ to $15.5 \mathrm{GHz}, \mathrm{S} 21$ is greater than $-2 \mathrm{~dB}$, and transparent rate of electromagnetic waves is greater than $63 \%$. Meanwhile, the bands in the range of $7.3-10.8 \mathrm{GHz}$ and $25.2-28.3 \mathrm{GHz}, \mathrm{S} 11$ and $\mathrm{S} 22$ are both below $-10 \mathrm{~dB}$, which means that the absorption rate in these two bands is larger than $80 \%$.

From Fig. 9(b), it can be seen that S12 is equal to S21 in Fig. 9(a). Nevertheless, the reflection coefficients are greater than $-10 \mathrm{~dB}$ out of its operating band. In some frequency points, it approximates $0 \mathrm{~dB}$. If the incident wave is along $\mathrm{Z}$ axis, it will get much more echo signal. To reduce RCS, the resistance layer of the radome is in the outside surface and the metal FSS layer is on internal surface.

The transmission coefficients under oblique incident angles for TE and TM polarizations are also simulated and can be seen in Fig. 10. The absorption rate is calculated by $1-S_{21}^{2}-S_{11}^{2}=10 \log _{10}\left(1-\left(10^{\left(S_{21} / 20\right)}\right)^{2}-\left(10^{\left(S_{11} / 20\right)}\right)^{2}\right) \mathrm{dB}$.

It can be seen that the proposed FSS demonstrates a stable frequency response under oblique incident angles

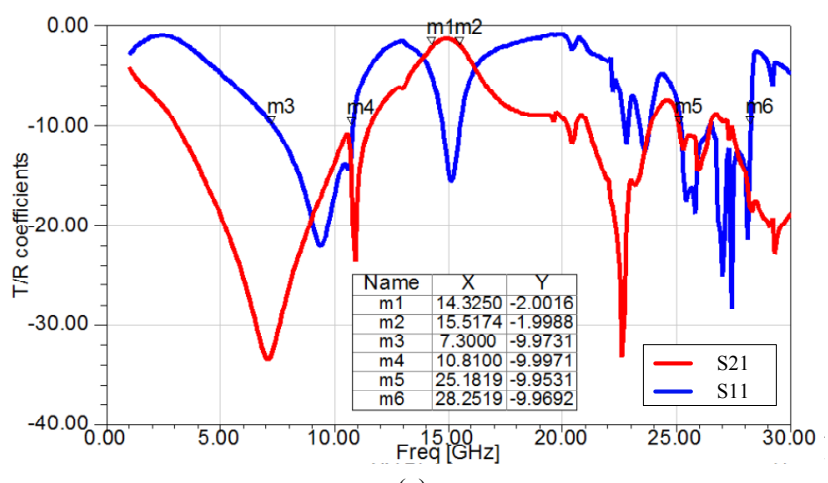

(a)

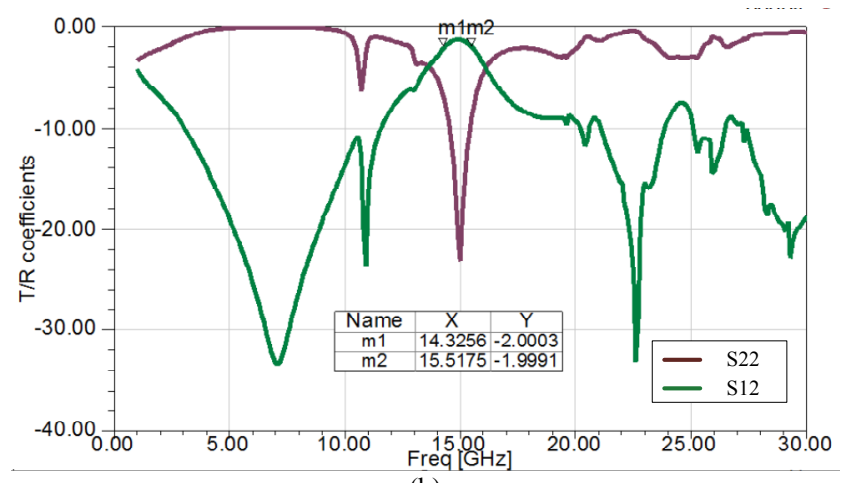

(b)

Fig. 9. The transmission and reflection parameters of designed FSS: (a) of incident wave along $-Z$ axis; (b) of incident wave along $+\mathrm{Z}$ axis. 


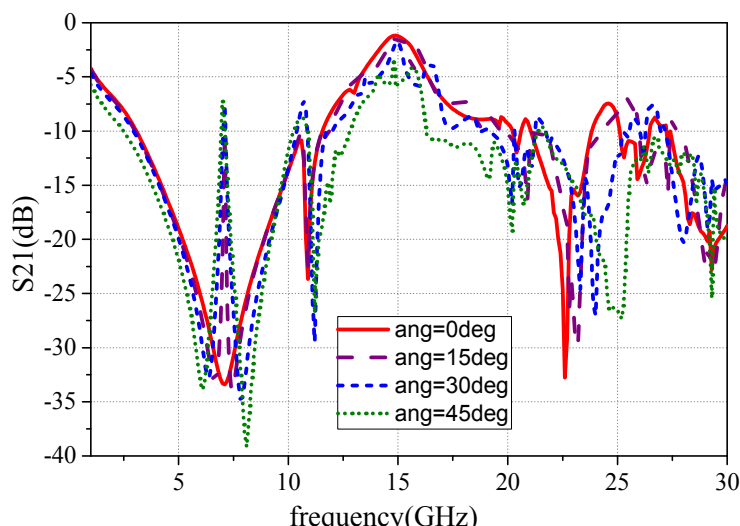

(a) S21 for TE polarization.

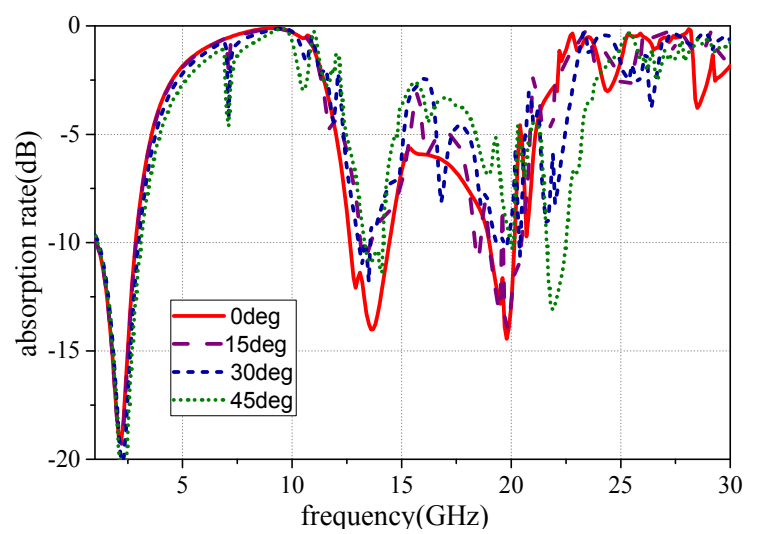

(b) Absorption rate for TE polarization.

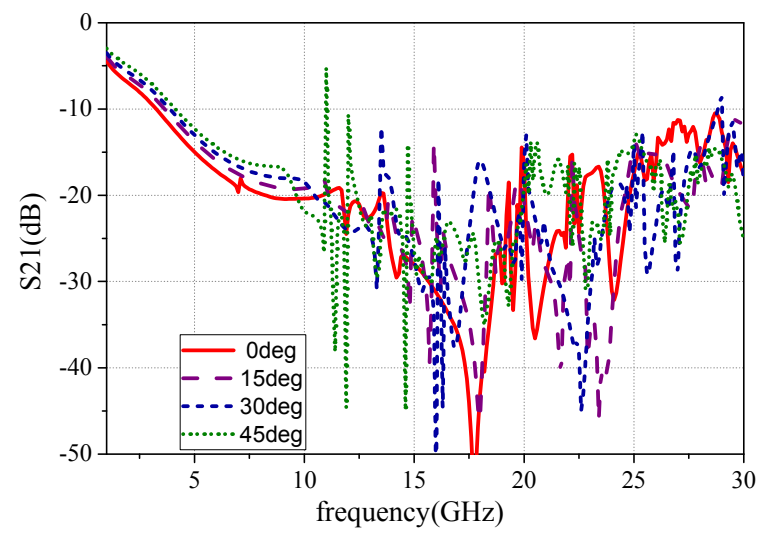

(c) S21 for TM polarization.

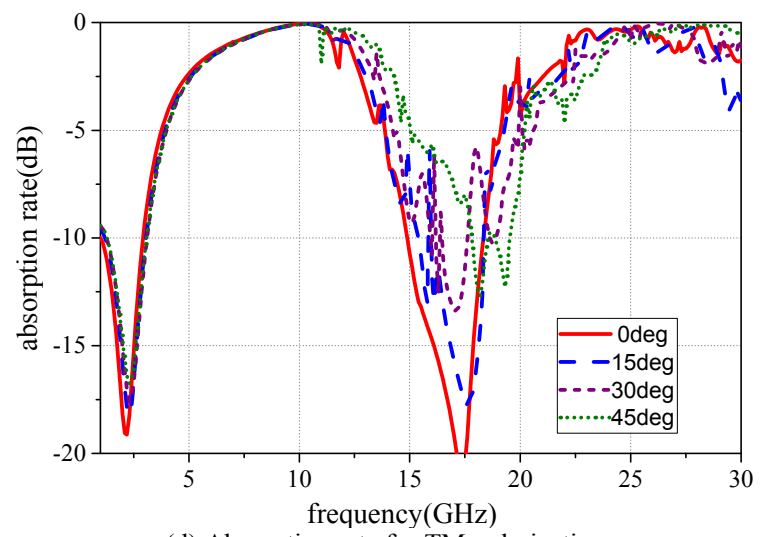

(d) Absorption rate for TM polarization.

Fig. 10 The transmission and absorption parameters of designed radome under oblique incident angles.

\begin{tabular}{|l|c|c|c|}
\hline & Our design & Design [13] & Design [14] \\
\hline Layer Number & $2(3)$ & $3(5)$ & $4(9)$ \\
\hline Thickness & $5 \mathrm{~mm}$ & $>20 \mathrm{~mm}$ & $20 \mathrm{~mm}$ \\
\hline Passband Loss & $<2 \mathrm{~dB}$ & $<2.5 \mathrm{~dB}$ & $<2 \mathrm{~dB}$ \\
\hline $\mathrm{CFP}$ & $15 \mathrm{GHz}$ & $10 \mathrm{GHz}$ & $10 \mathrm{GHz}$ \\
\hline Passband Width & $1.2 \mathrm{GHz}$ & $3.5 \mathrm{GHz}$ & $4.1 \mathrm{GHz}$ \\
\hline $\begin{array}{l}\text { Absorption band } \\
\text { (GHz) }\end{array}$ & $7.3-10.8 ;$ & $5 ;$ & $2.4-3.5 ;$ \\
\hline $\begin{array}{l}\text { Polarization } \\
\text { insensitive }\end{array}$ & $25.3-28.3$ & $14.5-17$ & $15.2-17.1$ \\
\hline
\end{tabular}

Tab. 2. Comparison of our design with other two similar reported radomes.

ranging from $0^{\circ}$ to $30^{\circ}$ for TE polarization. As for TM polarization, there is no transmission band since the structure of our design is not symmetrical around zero point. This is to say, the radome is polarization sensitive. Meanwhile, as the incident angle increases, the pass-band bandwidth and higher absorption band decrease. In addition, when incident angle reaches up to $45^{\circ}$, the frequency response deteriorates severely for TE polarization. Insertion loss in pass-band becomes larger while the out-of-band absorption gets worse. For TM polarization, out of band absorption is better than TE polarization in higher frequency bands and becomes more stable when incident angle increases. With no transmission band, the radome was shielding for unwanted incident TM polarization waves.

To better demonstrate the performance of the proposed structure, the designs in [13] and [14] which were made of resistance material and had two absorption bands are included for comparison in Tab. 2. In Tab. 2 CFP is short for center frequency of passband. The layer numbers only contain the periodic layers. The numbers in brackets contain dielectric substrate. Despite its polarization sensitive property and a bit narrow passband which can satisfy our target antenna, our design shows its obvious advantages in the thickness and wideband adsorption.

\section{Antenna and Radome System}

For further analysis of the performance of the designed radome, a radome covering on a waveguide slot antenna, which is the one to reduce the RCS, is as shown in Fig. 11. The planar size of this antenna is $78 \mathrm{~mm} \times 78 \mathrm{~mm}$.

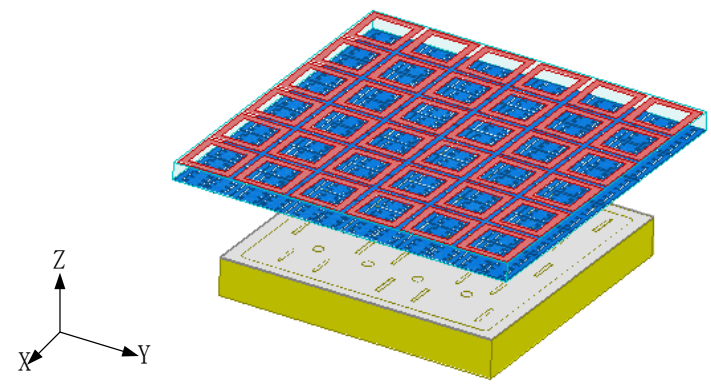

Fig. 11. The radome with wave-guide slots antenna. 
Limited by the size of the antenna, the size of the radome is designed to be $96 \mathrm{~mm} \times 96 \mathrm{~mm}$ ( 6 units $\times 6$ units).

\subsection{Effect on Antenna Gain}

The antenna working frequency is around $15 \mathrm{GHz}$, which is our target frequency. The radiation patterns of the antenna and the antenna with our radome are shown in Fig. 12. From the two figures, it can be found that the gain in main lobe of the antenna is almost not affected.

For detailed and clear comparison, the gains of the antenna and the covering radome in the band from $13 \mathrm{GHz}$ to $17 \mathrm{GHz}$ are calculated respectively. The influence of the radome on the gain of the antenna is less than $2 \mathrm{~dB}$, which can be neglected. In conclusion, the invisible radome does not affect the normal operation of the antenna.

\subsection{Effect on RCS of Antenna}

The main purpose of designing the invisible radome is to absorb the electromagnetic wave out of its working band. To proceed further, some simulations are done to verify that the radome made of this metamaterial can re-

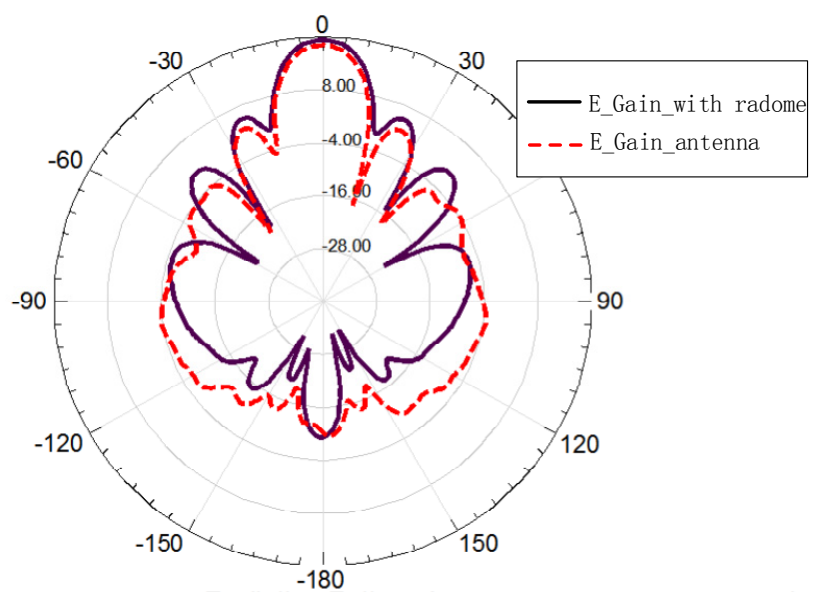

(a)

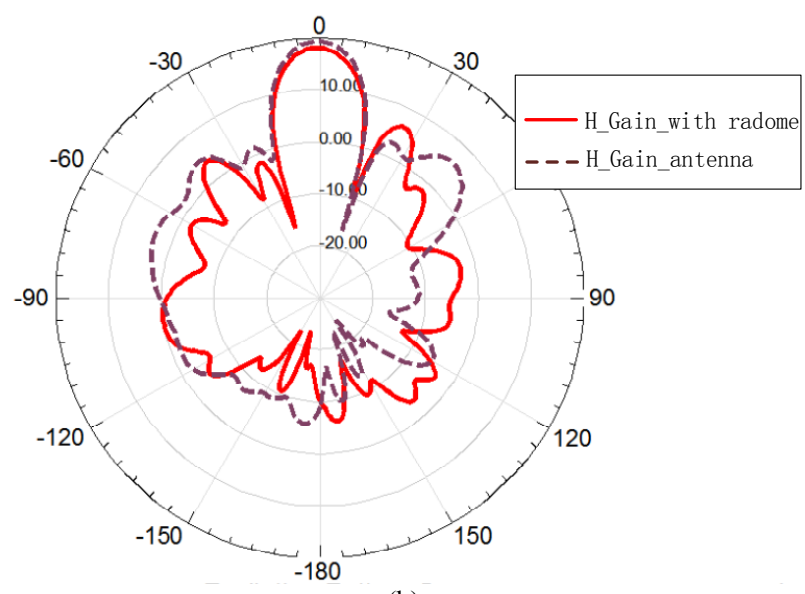

(b)

Fig. 12. (a) The E gain of the antenna and the antenna with radome. (b) The $\mathrm{H}$ gain of the antenna and the antenna with radome.

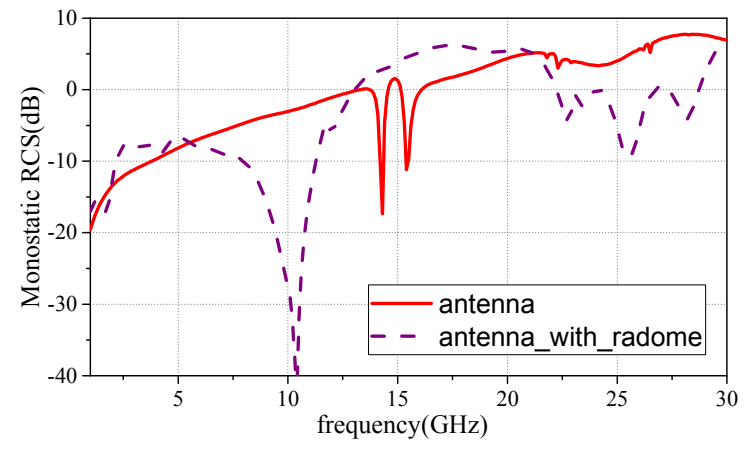

Fig. 13. The RCS of the FSS and the antenna.

duce the RCS. The antenna and the antenna with radome are put into an air box with radiation boundary separately. An incident plane wave with the propagation direction along $-\mathrm{Z}$ axis was set as excitation. After simulation, monostatic RCS of the antenna and the antenna with radome are given, as shown in Fig. 13.

The size of the FSS radome is $96 \mathrm{~mm} \times 96 \mathrm{~mm}$, while the size of the antenna is $78 \mathrm{~mm} \times 78 \mathrm{~mm}$. RCSs of the two objects are shown in Fig. 13. It is obvious that the size of the invisible radome is larger than that of antenna. However the RCS is at least $10 \mathrm{~dB}$ lower than the antenna around $10 \mathrm{GHz}$ and $26 \mathrm{GHz}$, respectively. In addition, the RCS will be reduced much more if the size of the FSS is nearly the same with the antenna. Consequently, the designed radome is feasible and effective.

\section{Conclusion}

A novel thin radome made by metamaterial with sandwich-like properties is presented. Its two absorption bands were separated by one transmission band, which is more attracting for radar stealth technology. Also, this new radome is made of three layers and its absorption layer is made of resistance film for easy fabrication, which can be more attracting in practical radome. To understand the operating principle, the absorption property is analyzed, and the goblet metal FSS is discussed. The qualitative analysis is given by an equivalent circuit model, and the main parameters are shown. The simulation results show good transmission property in the antenna working band and good RCS reduction ability bilaterally out of the work band.

\section{Acknowledgments}

This work was supported by the National Natural Science Foundation of China under Grant No. 61302017.

\section{References}

[1] ARCENEAUX, W. S., AKINS, R. D., MAY, W. B. Absorptive/transmissive radome. US Patent 5,400,043, 1995. 
[2] COSTA, F., MONORCHIO A. A frequency selective radome with wideband absorbing properties. IEEE Transactions on Antennas and Propagation, 2012, vol. 60, no. 6, 2012, p. 2740-2747. DOI: 10.1109/TAP.2012.2194640

[3] MONORCHIO, A., COSTA, F., MANARA, G. Efficient analysis of frequency-selective surfaces by a simple equivalent-circuit model. IEEE Antennas and Propagation Magazine, 2012, vol. 54, p. 35-48. DOI: 10.1109/MAP.2012.6309153

[4] COSTA, F., MONORCHIO, A., MANARA, G. An overview of equivalent circuit modeling techniques of frequency selective surfaces and metasurfaces. Applied Computational Electromagnetics Society Journal, 2014, vol. 29, no. 12, p. 960-976.

[5] LI WEN-HUI, ZHANG JIE-QIU, QU SHAO-BO, et.al. Radar cross section reduction of microstrip antenna based on wide-band metamaterial absorber. Acta Physica Sinica, 2015, vol. 64, no. 8, p. 084101(7). DOI: 10.7498/aps.64.084101 (in Chinese)

[6] GENOVESI, S., COSTA, F., MONORCHIO, A. Wideband radar cross section reduction of slot antennas arrays. IEEE Transactions on Antennas and Propagation, 2014, vol. 62, no. 1, p. 163-173. DOI: $10.1109 /$ TAP.2013.2287888

[7] LI SIJIA, CAO XIANGYU, GAO JUN, et.al. Design of ultra-thin broadband metamaterial absorber and its application for RCS reduction of circular polarization tilted beam antenna. Acta Physica Sinica, 2013, vol. 62, no. 12, p. 124101(10). DOI: 10.7498/aps.62.124101 (in Chinese)

[8] JIE ZHOU, SHAOWEI BIE, DONG WAN, et.al. Realization of thin and broadband magnetic radar absorption materials with the help of resistor FSS. IEEE Antennas and Wireless Propagation Letters, 2015, vol. 14, p. 24-27. DOI: 10.1109/LAWP.2014.2349533

[9] HUI YICONG, WANG CHUANQI, HUANG XIAOZHONG Design and fabrication of broadband radar metamaterial absorber based on the resistor FSS. Acta Physica Sinica, 2015, vol. 64, p. 218102(5). DOI: 10.7498/aps.64.218102 (in Chinese)

[10] ZHOU HANG, YANG LIWEI, QU SHAOBO, et.al, Experimental demonstration of an absorptive/transmissive FSS with magnetic material. IEEE Antennas and Wireless Propagation Letters, 2014, vol. 13, p. 114-117. DOI: 10.1109/LAWP.2013.2296992

[11] CHEN QIANG, FU YUNQI. A planar stealthy antenna radome using absorptive frequency selective surface. Microwave and Optical Technology Letter, 2014, vol. 56, no. 8, p. 1788-1792. DOI: $10.1002 / \mathrm{mop} .28442$

[12] YUPING SHANG, ZHONGXIANG SHEN, SHAOQIU XIAO. Frequency-selective rasorber based on square-loop and crossdipole arrays. IEEE Transactions on Antennas and Propagation, 2014, vol. 62, no. 11, p. 5581-5589. DOI: 10.1109/TAP.2014.2357427

[13] XUJIN YUAN, XIAOFENG YUAN. A transmissive/absorbing radome with double absorbing band. Microwave and Optical Technology Letters, 2016, vol. 58, no. 8, p. 2016-2019. DOI: $10.1002 / \mathrm{mop}$

[14] WEIWEI WU, TIANZHEN MENG, HUANG JINGJIAN, et.al. Study of a metamaterial with single passband between two neighboring absorptive bands. In $10^{\text {th }}$ European Conference on Antennas and Propagation. Davos (Switzerland), 2016, p. 1-4. DOI: 10.1109/EuCAP.2016.7481634

\section{About the Authors ...}

Tianzhen MENG was born in 1989. She received the B.S. degrees in Electrical and Information Engineering from Nanjing University of Aeronautics and Astronautics in 2012 and M.S. degree from Mechanical Engineering College in 2014. She is currently working toward the Ph.D. degree in Electronic Engineering from the National University of Defense Technology. She is interested in electromagnetic theory and metamaterial radomes. 